\title{
Las acciones del gobierno para los menonitas de una colonia en México
} Government actions for the Mennonites of a colony in Mexico As ações do governo para os menonitas de uma colônia no México

\author{
Marisol Cruz Cruz \\ marisol_cruz_cruz@yahoo.com.mx \\ Recibido: 6 de junio 2016 \\ Aprobado: 3 de octubre 2016
}

\section{Resumen}

Se exponen las acciones del gobierno para los menonitas en lo religioso y lo económico. El trabajo se divide en cuatro apartados. Para ello se aborda la perspectiva de las políticas públicas, entendidas como acciones implementadas por el gobierno en sus tres niveles para darle vida económica a la sociedad. Luego los materiales y métodos, que incluye la revisión de otras investigaciones, el rescate de la información de un diario de campo de 391 páginas, el surgimiento de expresiones y la relación de éstas para comprender las acciones del gobierno con la comunidad menonita. Los hallazgos sobre las acciones del gobierno con la religión y la economía menonita, nos remiten a su origen, su constante movilidad de Holanda hasta México, las estrategias específicas diseñadas para los menonitas por el gobierno con Chihuahua viven con los menonitas. Se trata de una relación de mutuo beneficio. Los menonitas buscan la preservación de su fe y el gobierno la dinámica económica.

Palabras clave: políticas públicas, menonitas, México 


\section{SIXO ${ }^{\text {? }}$ volumen 9, Números 1, 2015}

\begin{abstract}
The aim of this research is the expose overnment actions to Mennonites in religion and economics. The document observes the public policy, as actions by the government to give economic life of society. Then the materials and methods, including review of other research, rescue information from a field journal of 391 pages, to understand the actions of the government with the Mennonite community. The findings on the government's actions with religion and the Mennonite economy, remind us of their origin, their constant migration of Holland to Mexico, specific strategies designed to Mennonites by the government in Chihuahua lives with the Mennonites. It is a relationship of mutual benefit. Mennonites seek to preserve their faith and government economic dynamics.
\end{abstract}

Keywords: public policies, Mennonites, Mexico

\section{Resumo}

Apresentam-se as ações do governo para os menonitas nos aspectos religioso e econômico. $\mathrm{O}$ trabalho se divide em quatro seções. Para isso se aborda a perspectiva das políticas públicas, entendidas como ações implantadas pelo governo nos seus três níveis para dar vida econômica à sociedade. A seguir os materiais e métodos, que incluem a revisão de outras investigações, o resgate da informação de um diário de campo de 391 páginas, o surgimento de expressões e sua relação para compreender as ações do governo junto à comunidade menonita. As descobertas sobre as ações do governo com a religião e economia menonita, remetem-nos à sua origem, sua constante mobilidade de Holanda até o México, as estratégias específicas desenhadas para os menonitas pelo governo como Chihuahua vivem com os menonitas. Trata-se de uma relação de mútuo benefício. Os menonitas buscam a preservação de sua fé e o governo a dinâmica econômica.

Palavras chave: políticas públicas, menonitas, México

\section{Introducción}

La tesis de ésta investigación es que las prácticas del gobierno dirigidas a los menonitas tanto en lo religioso como en lo económico, tienen un efecto no buscado: el éxito económico de los lugares donde se asientan respecto a las comunidades vecinas de los nativos, es decir, la religión significa los 
elementos económicos, lo que explica la abundancia en una comunidad menonita.

Desde su origen los menonitas y el gobierno o los gobiernos (absolutistas, monárquicos, democráticos, progresistas), han establecido una relación de mutuo beneficio. Los menonitas lo hacen para la preservación de su fe y los gobiernos han buscado dinamizar la economía con las aportaciones de este grupo poblacional.

De la relación del gobierno con los menonitas se obtienen dos tipos de beneficio. Un beneficio positivo, porque se generan las condiciones para poder vivir en diferentes zonas impactando en lo económico; por ejemplo, la comercialización se facilita por la novedad de los productos, además de modernizar los procesos productivos. También se tiene un beneficio negativo, porque en los procesos económicos en que interviene el gobierno más que dinamizar la economía, la entorpece.

Los menonitas son considerados como protestantes por haber seguido una línea alterna a las reformas de la Iglesia católica realizada en 1521 e impulsada por Martín Lutero. Tienen como base de toda su sociedad la interpretación de la Biblia. Se bautizan en edades adultas, cuando ya son conscientes de su naturaleza pecaminosa, y se han arrepentido y han realizado la conversión. Los menonitas no creen en la transustanciación, consideran a Dios como omnipotente y creen en la trinidad (Padre, Hijo y Espíritu Santo) (Cid y Riu).

En los países donde habitan se asientan bajo una serie de consideraciones (conocidas como privilegium), otorgadas por los gobiernos (decreto presidencial en el caso mexicano), que les permite establecerse en determinadas zonas, vivir bajo su propia religión, su idioma, su educación, bajos sus propias reglas escritas y no escritas sin participación en asuntos del gobierno (tienen 
prohibido ser funcionarios o prestar servicio militar), no rendirle honores al lábaro patrio pero con pleno respeto y obediencia a las leyes de los gobiernos, por quienes oran para que conduzcan de la mejor manera los destinos de las sociedades.

En los espacios donde viven, se adaptan e innovan los procesos productivos. Así como Los Países Bajos volvieron productivas tierras no aptas para los cultivos y en Rusia se convirtieron en propietarios y productores de amplias extensiones de tierra, en Chihuahua se han convertido en uno de los principales productores y proveedores de maíz. En tanto, la zona de La Honda, Zacatecas, la han convertido en un eje dinamizador de la producción y comercialización primaria de maíz, frijol, avena y alfalfa, industria láctea. Además, se dedican a producir maquinaria e implementos agropecuarios.

Éste trabajo se aborda desde la perspectiva de las políticas públicas, entendidas como acciones implementadas por el gobierno en sus tres niveles para darle vida económica a la sociedad menonita. Luego dentro de los materiales y métodos se encuentran: esto es la revisión de otras investigaciones, el rescate de la información de un diario de campo de 391 páginas, el surgimiento de expresiones y la relación de las mismas para comprender las acciones del gobierno con la comunidad menonita.

La investigación es parte de un trabajo más amplio sobre ética religiosa y cultura económica de los menonitas en una localidad de México, cuyo objetivo es conocer la relación entre la religión y la economía, así como los procesos que dieron origen a esta relación y sus modificaciones hasta la actualidad, que la han permitido convertirse en una comunidad económicamente exitosa respecto a otras localidades vecinas. 


\section{La perspectiva de las políticas públicas}

Las políticas públicas son un conjunto de acciones que emprende el Estado para atender problemas públicos de diversas sociedades. Su origen se remonta a la década de los cincuenta en Estados Unidos y se ha extendido a todas las sociedades latinoamericanas (Dávila, Hurtado y Soto).

En México las acciones estatales las podemos ubicar una vez que concluyó la Revolución Mexicana. A partir de entonces, se emprendieron una serie de acciones encaminadas a reconstruir el país que había sido devastado y requería el impulso económico necesario para incentivar el mercado interno.

La acción estatal en México se ha enfatizado conforme han pasado los años, porque ha mantenido una presencia permanente en la solución de problemas públicos. Incluso se considera que posee la capacidad administrativa adecuada para solucionar las complicaciones que afectan a la sociedad, para lo cual se acuñó el término políticas públicas por Laswell en el texto The Policy Sciencies (Laswell y Lerner, 1951), en la idea de generar conocimiento de problemas públicos desde esta perspectiva, vinculando el estudio a actores y reglas del juego (Dávila, Hurtado y Soto).

Hablar de políticas públicas requiere de la reflexividad. Desde la conciencia del investigador, de su relación con la situación, volver sobre sí mismo para examinar críticamente el efecto que tiene su punto de vista, sus creencias académicas, políticas y sociales sobre el estudio, asumiendo la función de explicar un punto de vista y su institucionalización en prácticas y marcos de interacción (Roth).

El estudio orientado a los problemas públicos socioeconómicos debe ser estudiado en dos sentidos, de arriba hacia abajo y de abajo hacia arriba, esto es, tener una mirada de ambas partes para 
saber de la efectividad de las políticas públicas, en el que se da una relación del gobierno consigo mismo y con la sociedad (Roth).

\section{Materiales y métodos}

Se hace un recorrido histórico del origen y la actualidad de los menonitas, mediante la revisión de otras investigaciones que contienen elementos que permiten hacer inferencias sobre el proceso histórico de la relación de los menonitas con los gobiernos o en su caso, las acciones del gobierno con la religión y la economía de los menonitas. De esos documentos y de un diario de campo de 391 páginas, se tomaron expresiones vinculadas o relacionadas al gobierno, la religión y la economía de los menonitas, para comprender las acciones del gobierno con la comunidad menonita de arriba hacia abajo y de abajo hacia arriba, con intereses totalmente contrapuestos. Los menonitas buscan mantener su fe y obras y el gobierno dinamizar la economía, que es un efecto no buscado por los menonitas.

\section{Análisis y discusión: la relación histórica de los menonitas con el gobierno}

Los menonitas son considerados una población ermitaña, pacifista (Valadéz) que históricamente ha sido una población migrante por diversos factores, entre ellos, la persecución, las restricciones a la adquisición de terrenos, el debilitamiento a su liderazgo y la pérdida de su capacidad para administrar sus propias escuelas están vinculadas a las acciones implementadas por los gobiernos de cada nación habitada, pues estos les retiraron el privilegium, una serie de consideraciones particulares, que incluye la libertad para practicar su religión, la exención del servicio 
militar, y el derecho de tener sus propias escuelas en las que el alemán fuera el idioma de enseñanza.

Los primeros movimientos poblacionales realizados indican que la migración de los menonitas fue entre zonas rurales de Europa. A mediados del siglo XVI llegaron a radicar en Polonia, concretamente de la región de Danzig, en Los Países Bajos, donde permanecieron de 1548 a 1572 bajo el reinado de Sigismund II, quien fue deslumbrado por su habilidad para mantener productivas tierras no aptas para los cultivos, “... ganar terreno por medio de la desecación del suelo (aprendido en Holanda) y con ello lograron se les concediera el privilegium" (Tylor). Sin embargo, tuvieron que migrar por un reajuste territorial de Polonia.

De esta manera llegaron a Rusia en 1786, pero en 1870 el gobierno de aquel país publicó un decreto que dio por finalizada la administración de las colonias extranjeras y los menonitas quedaron sujetos al servicio militar debilitándose; además, tuvieron restricciones para adquirir tierras. Dicha situación generó una movilidad poblacional, hacia un lugar que les otorgará el privilegium. Frente a la negativa de Estados Unidos, apareció el gobierno de Canadá.

... en 1869 aceptó firmar un arreglo con los menonitas para apoyar la colonización de nuevas tierras... entregándoles en 1873 dos reservas: la Reserva del Este y la Reserva del Oeste, con más de 500 mil hectáreas de terrenos a bajo precio, ubicado en los dos lados del río Rojo de la provincia de Manitoba. (Tylor)

La Primera Guerra Mundial generó un ambiente hostil para los menonitas en Canadá, además el gobierno incursionó en su sistema educativo y los precios de los bienes agrícolas cayeron tras la Primera Guerra Mundial. Esto orilló a los menonitas a migrar a nuevos países donde habitar, siempre que se les garantizara 
el privilegium. Así viajaron a Brasil y Argentina, pero el ambiente propicio lo encontraron en México.

Los Altkolonier de la región de Hague-Osler, Saskatchewan, hicieron un contacto con el empresario Arturo J. Braniff, cuñado del presidente Álvaro Obregón, y platicaron sobre la posibilidad de poder adquirir terrenos y un privilegium en México, porque consideraban preferible mudarse a México, en caso de conseguir un privilegium, que quedarse en Canadá. Alentados por los resultados de su entrevista, la comisión de los menonitas envió una carta a la Secretaría de Relaciones Exteriores en el que dieron una explicación detallada sobre los motivos de los menonitas para migrar a México y los beneficios que podrían aportar al país. En 1921 una delegación de menonitas partió a México, el 28 de enero llegó a El Paso, el 3 de febrero la comitiva entró a México por Nogales, inspeccionaron algunas posibilidades de compra en Sonora, Sinaloa, Nayarit y Jalisco. El 17 de febrero, el grupo se entrevistó con Álvaro Obregón para discutir los motivos de migrar a México y la posibilidad del otorgamiento de un privilegium. El presidente aceptó y a pesar de la corriente de nacionalismo y anti-extranjerismo que había surgido en el transcurso de la lucha armada, se logró el consenso entre los jefes revolucionarios sobre los beneficios de la migración extranjera. (Tylor)

El ambiente para arribar al país era propicio porque México estaba saliendo de la Revolución Mexicana, de la que emanó el reparto agrario para la gran masa que había participado activamente en el movimiento. También había surgido la tarea impostergable de impulsar un país progresista, por lo que el gobierno justificó la llegada de extranjeros, pues consideraba que el país era uno de los más ricos de la Tierra, pero tenía pocos habitantes. Aunque tenía "suficientes recursos para darles de comer y vestir a cien millones de habitantes, no se ha podido vestir a dos millones y el resto de ellos andan semidesnudos", por lo tanto, para 
resolver esa dicotomía, de acuerdo a Álvaro Obregón, se requería el capital extranjero para desarrollar todas las riquezas (Tylor).

Demográficamente la República Mexicana se percibía como un país inmenso para los escasos sujetos que lo habitaban, por lo que era necesario tomar acciones para poblarlo, así que se emitieron instrumentos legales para resolver el problema. Según el gobierno, una solución era a través de la colonización, para lo cual emerge la Ley sobre colonización y deslinde de terrenos baldíos el 15 de diciembre de 1883, promulgada por Manuel González, con la cual se mandó deslindar, medir, fraccionar y valuar los terrenos baldíos o de propiedad nacional de 2.500 hectáreas para cederlos a los inmigrantes extranjeros y habitantes de la República Mexicana que desearan establecerse como colonos en el país, gozarán por 10 años de diversas exenciones (Barragán). De ahí emanaron los beneficios para los menonitas.

Así, en febrero de 1921, Álvaro Obregón suscribió el documento por el que su gobierno se comprometió a respetar y garantizar a los menonitas el privilegium:

1) no estarán ustedes obligados al servicio militar; 2) en ningún caso se les obligará a prestar juramento; 3) tendrán el derecho más amplio de ejercitar sus principios religiosos y practicar las reglas de su Iglesia, sin que se les moleste o restrinja en forma alguna; 4) quedan ustedes plenamente autorizados para fundar sus propias escuelas, con sus propios maestros, sin que el gobierno los obstruccione en forma alguna; 5) por lo que se refiere a este punto, nuestras leyes son ampliamente liberales, podrán ustedes disponer de sus bienes en la forma que lo estimen conveniente y este Gobierno no presentará objeción alguna a que los miembros de sus secta establezcan entre ellos mismos el régimen económico que voluntariamente se propongan adoptar. (Barragán) ${ }^{1}$

1 Este documento del Presidente Álvaro Obregón fue dirigido a los representantes de la Old Colonia Reinland Mennonite Church, Rev. Julius Lowen, Johann Loeppky, 
Garantizado el privilegium por parte del gobierno mexicano, los menonitas adquirieron 112 mil hectáreas de tierra en suelo mexicano que les vendió Pedro Zuloaga, antes de ser obligado a venderlas ante las demandas agrarias del Estado o ser repartidas entre la masa participante en la Revolución Mexicana. Entre "1922-1926 unos 36 trenes llenos de menonitas procedentes de Saskatchewan y Manitoba, colonias establecidas en Canadá, con todo lo que disponían en casa, implementos agrícolas y formas de vida, llegaron a establecerse a San Antonio de los Arenales (hoy Ciudad Cuauhtémoc, Chihuahua) y se asentaron en los valles de Bustillos y Santa Clara. La segunda concentración más grande es la del estado de Durango, donde se asentó la más conservadora de las colonias: Old Colony. Años después, se establecieron en Zacatecas" (Barragán, 1996, 40-41).

La Gran Depresión junto con los controles impuestos por el gobierno mexicano sobre su ingreso al país ocasionó un descenso de los proyectos colonizadores de los menonitas. El estallido de la Segunda Guerra Mundial y la fobia hacia los menonitas resurgieron, por lo que se detuvo por un tiempo la migración de los menonitas. Se supone que los menonitas enseñarían a los agricultores locales todas sus novedades para mejorar la producción, pero la relación entre los nativos y los colonizadores no era del todo armoniosa.

El cobijo del gobierno mexicano con los menonitas permaneció y eso les permitió realizar su segunda movilidad poblacional interna en 1964, de Durango a las comunidades de La Honda en Miguel Auza y La Batea en Fresnillo, Zacatecas. De La Honda se han movilizado a Campeche, Monclova en Coahuila y América del Sur, concretamente en Paraguay, donde han fundado

Gerente Benjamín Goertzen y miembros como Cornelius Rempel, Klass Heide y David Rempel, en contestación a su ocurso de enero 29 del actual (1921) en el cual expresan el deseo de venir a establecerse como colonos agrícolas. 
la Colonia Durango (Barrgán, 1996: 40-41), rigiéndose con el privilegium traducido en el decreto presidencial emitido por Álvaro Obregón en 1921.

En los últimos años del siglo XX también se han asentado en Yucatán, Tamaluipas, San Luis Potosí, Sonora y Sinaloa.

Si bien el Estado mexicano ha implementado acciones para el asentamiento de los menonitas en México, es en el 2011 que se implementa un programa de política pública específico para esta población denominado Chihuahua Vive con los Menonitas, pensada, diseñada e implementada para los menonitas por el gobierno estatal.

Esta estrategia se ha establecido a partir de considerar a la comunidad menonita como pilar esencial que ha impulsado a la economía de la región noreste de la entidad en las zonas de Cuauhtémoc, Riva Palacio, Namiquipa y Cusihuiriachi, con una población aproximada de 38.000 habitantes, Ascensión, Buenaventura, Nuevo Casas Grandes, Janos y Villa Ahumada, con 7.110 habitantes y Ojinaga, Aldama con 1.918 pobladores.

Mediante el Programa Sectorial Chihuahua Vive con los Menonitas se han establecido diversas acciones para facilitar la interacción de la comunidad menonita con las diferentes dependencias del gobierno del Estado para darle vida económica a la sociedad.

El programa está enfocado en diseñar y fomentar programas para el desarrollo de la comunidad menonita, dar respuesta a la problemática y necesidades de la población menonita en un marco de respeto a su cultura y tradiciones, ya que se trata de una comunidad con un nivel de desarrollo social, económico, educativo y cultural, con amplia capacidad para gestionar ante las tres órdenes de gobierno sus necesidades y plantear su problemática 
con el propósito de impulsar una vida paralela y equitativa con las diferentes comunidades del territorio estatal.

El programa se aplica mediante la coordinación y compromiso de colaboración interinstitucional entre las diferentes dependencias, que abarcan lo económico, lo social, lo cultural, la educación, la salud, entre otros.

Como se observa, la relación que se ha establecido entre los menonitas y el gobierno es de abajo (los menonitas) hacia arriba (gobierno) y de arriba (gobierno) hacia abajo (menonitas), es decir, se ha conjuntado un proceso que ha sido visto por separado (de arriba hacia abajo y de abajo hacia arriba) (ver esquema 1) como sostienen Dávila, Hurtado y Soto sobre la evolución de las políticas públicas.

\section{Esquema 1.}

Menonitas y políticas públicas

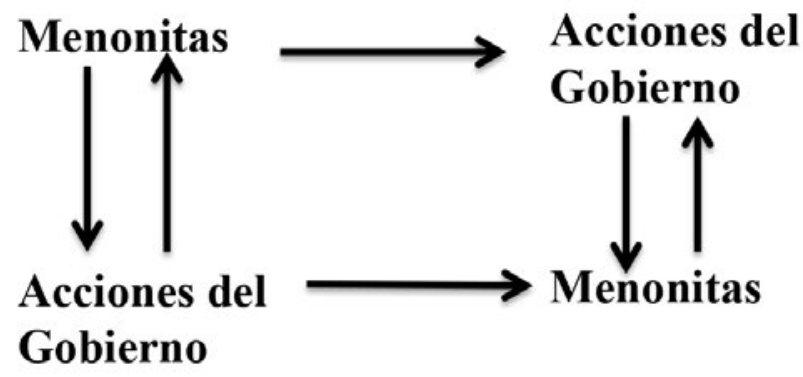

Fuente. Elaboración propia.

Es decir, primero, los menonitas asumen que existe una ley depositada en los gobernantes. A estas leyes. Ellos obedecen estas leyes, las respetan y tienen obligaciones con el gobierno, particularmente el pago de impuestos. Segundo, los menonitas no esperan prebendas, ni créditos, ni subsidios pero si el gobierno se 
los ofrece, ellos los aprovechan para sus procesos de producción y comercialización, ya que el flujo de recursos permite hacer el bien al pueblo de Dios que incluye a su comunidad cerrada y a la población del mundo abierto.

La relación de abajo hacia arriba o de los menonitas hacia el gobierno, se sintetiza en las expresiones: el privilegium, el pago de predial, la declaración de impuestos a la SHCP, y el pago de impuestos por consumo de bienes y servicios. Se trata de transferencias que realizan los integrantes de la sociedad menonita a los distintos niveles del gobierno.

De la relación de arriba hacia abajo o de las transferencias del gobierno a las familias menonitas, se sintetizaron las expresiones recibir subsidios (despensas o dádivas, diesel y semillas), recibir PROCAMPO, recibir créditos para la producción y/o la comercialización, que regularmente son créditos de peso por peso para los procesos de comercialización.

La banca privada también otorga créditos a tasas de interés establecidos por ella misma. Esto se sintetizó en la expresión recibir crédito privado.

La información disponible permite inferir que existe está relación primero de la sociedad menonita con el gobierno y luego del gobierno con los menonitas.

Es importante considerar la relación menonita-gobierno de esta manera, porque como sociedad se oponen a participar de manera activa en este nivel, a trabajar u ocupar algún cargo, pero aceptan la existencia de aparato estatal para el cobro de impuestos y aunque no es su propósito buscar financiamiento a sus procesos productivos y de comercialización, aceptan la oferta de los tres niveles de gobierno en cuanto a créditos a tasas bajas, 
peso por peso o fondo perdido porque asumen que son para las buenas obras del pueblo del Ser Supremo.

\section{A manera de conclusión}

La persecución por sus creencias religiosas llevó a los menonitas a entablar una relación con los distintos gobiernos como el monárquico, el absolutista, los democráticos y progresistas para llevar una vida en fe y obras, es decir, históricamente ha sido una relación de abajo hacia arriba, de la comunidad menonita al gobierno.

En la búsqueda de vivir en paz, como efecto no buscado, llamaron la atención de los gobiernos por su capacidad de adaptación a los procesos productivos y el mejoramiento de los mismos, particularmente la tierra.

Después de Canadá se vincularon con otros gobiernos en América Latina. En México se vincularon con el gobierno democrático de Alvaro Obregón.

La movilidad poblacional a México estuvo motivada por la necesidad de los menonitas de seguir contando con el Privilegium, pero el gobierno mexicano tenía la necesidad de reconstruir al país devastado por la Revolución. Así, los menonitas buscaban vivir con fe y obras, y el gobierno mexicano buscaba una mejora económica, poblar al país y mejorar la raza.

Aunque con algunos gobiernos vieron amenazado el privilegium y vivieron etapas de tensión por hurtos y casos de violación a mujeres menonitas, recibieron el respaldo del gobierno a través de acciones implementadas por las diversas instancias de seguridad para mantener la gobernabilidad en los asentamientos de los menonitas. 
A la par, por capacidad para innovar y mejorar la economía, los gobiernos continuaron con la implementación de estrategias para el asentamiento de los menonitas en distintos espacios geográficos del mundo.

El gobierno mexicano como otros de América Latina, generó condiciones para su establecimiento en el estado norte de Chihuahua, donde los menonitas viven de acuerdo a sus reglas de profesar su propia religión, dirigir su propia educación y dinámica económica.

En el caso mexicano, su establecimiento fue propicio porque se esperaba que contribuyeran a forjar una nación progresista, que estaba reconstruyéndose tras la devastación y destrucción de la Revolución Mexicana.

Por ello, mediante un Decreto Presidencial, en 1921 los menonitas adquirieron tierras para cultivos agrícolas que mejoraron la economía regional.

Pero es hasta el 2011 que el gobierno implementa el Programa Sectorial Chihuahua Vive con los Menonitas, que atiende tres rubros: social, cultural y económico, es decir, la relación de arriba hacia abajo, del gobierno hacia la comunidad menonita es reciente. Es con la implementación de este programa sectorial que se evidencia la relación del gobierno consigo mismo y de él con la sociedad.

Chihuahua es una excepción en el diseño, implementación y seguimiento de un programa sectorial, porque no hay indicios de que en otras entidades del país exista un programa público específico para los menonitas, que sólo buscan vivir con fe y obras.

En Zacatecas, al igual que en otras entidades donde residen los menonitas, las políticas públicas son de aplicación general y 
los nativos acceden a los programas sociales y económicos cubriendo los requisitos marcados en las reglas de operación.

\section{Bibliografía}

- Barragán, Patricia Verónica. «Menoniyas, etnicidad y derecho.» Serie de Cuadernos Jurídicos (1996).

- Cid, Carlos y Manuel Riu. Historia de las religiones. España: Óptima, 2000.

- Dávila, Mireya, Alberto Hurtado y Ximena Soto. «iDe qué se habla cuándo se habla de Políticas Públicas? Estado de la discusión y actores en el Chile del Bicentenario.» Revista Chilena de Administración Pública (2011): 5-33.

- Gobierno del Estado de Chihuahua. Programa Chihuahua Vive con los Menonitas. Líneas de acción. Chihuahua: Gobierno del Estadod de Chihuahua, 2012.

- Roth, Andrew. «Hacía una epistemología de las políticas públicas.» Revista Vetas de El Colegio de San Luis (2008): 13-29.

- Tylor, Lawrence Douglas. «Las migraciones menonitas al norte de México entre 1922 y 1940.» Revista Migraciones Internacionales (2005): 5-31.

- Valadéz, Alfredo. «Los menonitas de Zacatecas: de migrantes a empresarios exitosos.» La Jornada 08 de 05 de 2006: 32.

- Zapata, Oscar. Herramientas para elaborar tesis e investigaciones socioeducativas. México: Pax, 2005. 